\title{
Use of mechanical extraction devices in basilar artery occlusion
}

\author{
P Favrole, J P Saint-Maurice, M G Bousser, E Houdart
}

J Neurol Neurosurg Psychiatry 2005;76:1462-1464. doi: 10.1136/jnnp.2004.060491

Three consecutive patients with embolic basilar artery occlusion were treated with endovascular mechanical thrombus extraction. Recanalisation was rapidly obtained in one patient, who had a good initial recovery, and in another patient who made a complete recovery. By contrast, recanalisation failed in the third patient, who made no recovery. No haemorrhagic complications were detected. This technique may have advantages over thrombolysis in both efficacy and safety.

B asilar artery occlusion has a severe prognosis with a 75 $91 \%$ mortality in older series of patients treated only with anticoagulants. ${ }^{12}$ A decreased mortality of $55-70 \%$ has been reported in non-controlled series of patients treated with intra-arterial thrombolysis. ${ }^{3}$ In these series, outcome was mainly dependant on the success of recanalisation. ${ }^{3}$ In one recent report, distal territory involvement, embolism, and complete basilar artery occlusion were significant predictors of poor outcome. ${ }^{4}$ Furthermore, with successful recanalisation, there was a risk of haemorrhagic transformation (0 to $14 \%){ }^{3} \quad$ Endovascular mechanical thrombus extraction (EMTE) is currently being developed in an attempt to improve the recanalisation rate, while decreasing the haemorrhagic risk. ${ }^{5-11}$ We report three consecutive patients with embolic basilar artery occlusion who were treated with this technique.

\section{CASE 1}

A 73 year old man had a sudden right hemiparesis. He rapidly became tetraplegic with severe dysarthria (NIHSS: 25). Diffusion weighted magnetic resonance imaging (DWI) showed high signal intensities in the pons and cerebellar hemispheres, suggestive of multiple emboli. Magnetic resonance angiography (MRA) revealed a segmental flow void at the mid-part of the basilar artery. Cerebral angiography, begun 4.5 hours after clinical onset, showed an endoluminal defect at the mid-part of the basilar artery, with normal appearances proximal and distal to this defect. A goose neck snare, $4 \mathrm{~mm}$ in size (Microvena; fig $1 \mathrm{~B}$ ), was used to catch the thrombus. At the end of the procedure the basilar artery was completely recanalised, without residual stenosis. No haemorrhage was detected on computed tomography (CT) immediately after the angiographic procedure or 48 hours later. The patient recovered quickly in the first few days after the procedure (mild tetraparesis on day 12, NIHSS score 11). However, over the following days he developed septic shock from cholangitis associated with a pancreatic carcinoma and he died one month after the first symptoms.

\section{CASE 2}

A 39 year old woman developed a sudden left hemiparesis. Ten years earlier, she had undergone a mechanical aortic

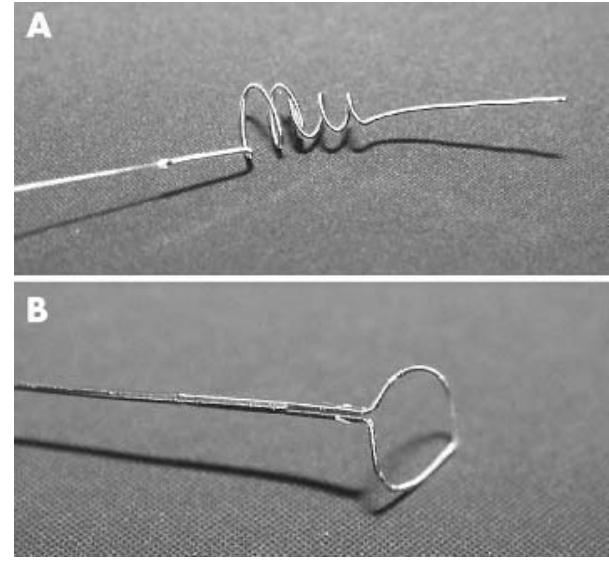

Figure 1 Examples of devices. (A) Concentric retriever (Concentric Medical). (B) Goose neck snare (Microvena).

valve replacement for rheumatic heart disease. She was treated with oral anticoagulants, but she had decided to stop this treatment one month before. One hour after the clinical onset, she was tetraparetic, drowsy, and required assisted ventilation (NIHSS score 25). Brain CT revealed a spontaneously hyperdense basilar artery. Cerebral angiography 12 hours after her condition worsened showed complete occlusion of the basilar artery at the level of the anteriorinferior cerebellar arteries. Both posterior cerebral arteries were filled by the carotid system through the posterior communicating arteries, but no retrograde flow to the basilar artery was seen. Despite several extraction attempts with mechanical devices (goose neck snares, 2 and $4 \mathrm{~mm}$ large (Microvena; fig 1B), In Time four fibre system (Boston Scientific), thromboaspiration with a Tracker 38 catheter), the thrombus could not be retrieved from the basilar artery. No local thrombolysis was attempted owing to the important delay between the worsening of neurological symptoms (tetraplegia) and the end of the endovascular procedure (more than 16 hours). Magnetic resonance imaging (MRI) showed a massive pontomesencephalic infarct without haemorrhage. There was no clinical improvement and the patient remained with a locked-in syndrome (modified Rankin scale score 5 at three months).

\section{CASE 3}

A 37 year old man had sudden occipital headache, with vomiting and dysarthria. Five hours later he worsened, developing a right hemiplegia. The initial brain CT revealed only a spontaneously hyperdense basilar artery. Four hours later he was tetraparetic, with drowsiness and dysphagia

Abbreviations: EMTE, endovascular mechanical thrombus extraction; $\mathrm{MERCl}$, mechanical embolus removal in cerebral ischaemia; NIHSS, National Institutes of Health stroke scale 

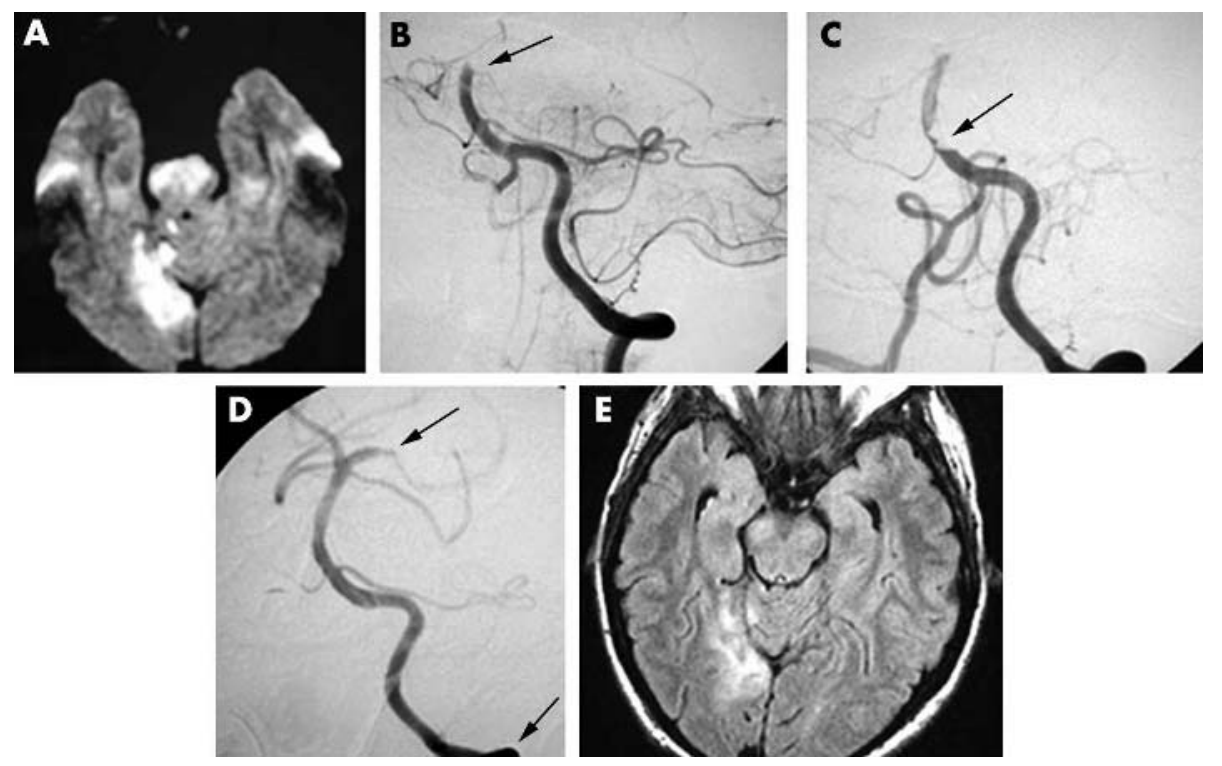

Figure 2 Case 3. (A) Initial diffusion weighted sequence showing acute pontine, cerebellar, and temporo-occipital infarcts. (B) Initial angiography of the left vertebral artery showing the occlusion of the top of the basilar artery and superior cerebellar arteries (arrow). (C) Control angiography after attempt of recanalisation with the Concentric retriever (Concentric Medical) showing persistent basilar artery occlusion, and mobilisation of the embolus in the middle part of the basilar artery with occlusion of the left anterior-inferior cerebellar artery. (D) Final control angiography after thrombus extraction with a goose neck snare, showing the complete basilar artery recanalisation and filling defects suggesting small distal emboli in the first segment of the left posterior communicating artery (arrow). (E) Flair sequence carried out one month after recanalisation showing only ischaemic sequelae in the right occipital lobe and in the right part of the vermis.

(NIHSS score 22) and was then transferred to our unit. MRI showed recent and multiple brain stem, cerebellar, and occipital infarcts (fig 2A). MRA revealed a segmental flow void in the distal basilar artery. Cerebral angiography, carried out 12 hours after the onset of symptoms and seven hours after worsening, showed occlusion of the top of the basilar artery and of the superior cerebellar arteries (fig 2B); there was no retrograde filling from the posterior communicating arteries. The first attempt with the Concentric retriever (Concentric Medical; fig 1A) failed to recanalise the artery and moved part of the thrombus which occluded the left anterior-inferior cerebellar artery (fig 2C). The entire thrombus was retrieved from the basilar artery with a $4 \mathrm{~mm}$ goose neck snare (Microvena; fig 1B). Complete recanalisation of the basilar artery, superior cerebellar arteries, and the left anterior-inferior cerebellar artery was achieved, but two filling defects suggesting small distal emboli were detected in the first segment of the left posterior communicating artery (fig 2D). No additional in situ thrombolysis was undertaken. Control CT at 24 hours showed no haemorrhage. The patient recovered rapidly and was completely asymptomatic three months later (modified Rankin scale score 0). Control MRI at one month showed small right posterior cerebral artery and partial right superior cerebellar artery infarcts (fig 2E). Despite detailed diagnostic workup no cause was found.

\section{DISCUSSION}

The outcome differed greatly in these three patients treated with EMTE for severe brain stem ischaemia caused by basilar artery occlusion. Recanalisation was rapidly obtained in patient 1 , who had a good initial recovery but who later died from pancreatic carcinoma, and in patient 3, who recovered remarkably well. By contrast, recanalisation failed in patient 2 , who made no recovery. Although the precise cause of the basilar artery occlusions was unknown, the absence of atheroma or of other varieties of arterial wall disease suggested cardiac emboli despite the absence of an identified cardiac source in patients 1 and 2 . The main difference between the three cases was the delay between the severe clinical impairment (tetraplegia or reduced consciousness) and the procedure-12 hours from onset in patient 2, contrasting with 3.5 and 4 hours, respectively, in patients 1 and 3. This is consistent with previous reported data suggesting that the sooner the intervention is initiated the higher the recanalisation rate, and the better the prognosis. ${ }^{12}{ }^{13}$ The absence of an effective collateral supply, the long clot length, and the proximal clot location are also probably partly responsible for the lack of recanalisation associated with the poor prognosis in patient 2, as suggested by others. ${ }^{14-17}$

EMTE may have advantages over thrombolysis with regard to both efficacy and safety. Studies with intra-arterial thrombolysis have shown that the outcome depends mainly on the recanalisation rate, ${ }^{3}$ which is low in large and proximal occlusions, such as extensive basilar artery occlusion or in internal carotid artery $\mathrm{T}$ occlusion (0 to $5 \%$ in larger $\left.\operatorname{series}^{18}{ }^{19}\right)$. Extraction of such large thrombi with mechanical devices is feasible and there are reported cases in which thrombolysis failed to lyse the clot, which was then caught by endovascular snares with partial or complete arterial recanalisation..$^{5}$

Furthermore, it is well established that the sooner the recanalisation is achieved, the better the clinical prognosis. EMTE can remove the clot very rapidly, as suggested by Wikholm ${ }^{7}$ who reported two cases of acute basilar artery occlusion in which angiographic diagnosis and thrombus extraction were achieved in only 15 and 20 minutes. Moreover if the snare fails, intra-arterial fibrinolysis can be started immediately because the microcatheter is already in place. The combined use of these two therapeutic methods has already been reported ${ }^{8}$ without haemorrhagic complication.

As regards safety, EMTE has the advantage over thrombolysis of being able to recanalise the artery without modifying haemostasis. It can thus be used in patients who have a contraindication to thrombolysis. In our patients, no parenchymal haemorrhagic complications were detected on the 
control brain imaging (CT or MRI) at 24 hours, in keeping with previously reported patients. ${ }^{7-9}$ It remains to be seen if these potential advantages are compatible with an increased therapeutic window over the present six hours accepted for intra-arterial thrombolysis in middle cerebral artery occlusion. ${ }^{20}$ Recent data from the MERCI trial suggest that EMTE carried out within eight hours of symptoms onset is safe, and that successful recanalisation could benefit a significant number of patients. ${ }^{21}$

On the other hand, EMTE has potential risks. The first is fragmentation of the thrombus with occlusion of distal arteries either by the thrombus itself or by emboli. This occurred in patient 3, in whom the first recanalisation attempt moved part of the thrombus which occluded the left AICA and the second was successful with regard to recanalisation but was accompanied by two small left posterior cerebral artery emboli. Despite these complications the patient made a complete recovery. The control MRI showed no additional ischaemic lesion in these territories. These complications appear to be mainly technical; the solution requires either the use of a system that will definitely catch the thrombus, or flow control (as recommended by Mayer $e t a l^{8}$ ). The second risk is vascular trauma with perforation or dissection, which did not happen in our three patients.

These cases show that EMTE is feasible in basilar occlusion and that when recanalisation is obtained early after the onset of symptoms, it is compatible with an excellent neurological recovery. Large studies are needed to evaluate the benefit to risk ratio of EMTE and the combination with intra-arterial thrombolysis in basilar artery occlusion. The combination of these endovascular techniques also needs to be compared with intravenous thrombolysis in basilar artery occlusion. ${ }^{22}$

\author{
Authors' affiliations \\ P Favrole, M G Bousser, Department of Neurology, Hôpital Lariboisière, \\ Paris, France \\ J P Saint-Maurice, E Houdart, Department of Neuroradiology, Hôpital \\ Lariboisière \\ Competing interests: none declared \\ Correspondence to: Dr P Favrole, Department of Neurology, \\ Lariboisiere Hospital, 2 rue Ambroise Paré, 75475 Paris, France; \\ pascal.favrole@lrb.ap-hop-paris.fr \\ Received 7 December 2004 \\ In revised form 31 January 2005 \\ Accepted 2 February 2005
}

\section{REFERENCES}

1 Archer CR, Horenstein S. Basilar artery occlusion. Stroke 1977;8:383-90.

2 Hacke W, Zeumer $\mathrm{H}$, Ferbert A, et al. Intra-arterial thrombolytic therapy improves outcome in patients with acute vertebrobasilar occlusive disease. Stroke 1988; 19:1216-22.

3 Brandt T. Diagnosis and thrombolytic therapy of acute basilar artery occlusion: a review. Clin Exp Hypertens 2002;24:611-22.

4 Voetsch B, DeWitt LD, Pessin MS, et al. Basilar artery occlusive disease in the New England Medical Center Posterior Circulation Registry. Arch Neurol 2004;61:496-504.

5 Chopko BK, Kerber C, Wong W, et al. Transcatheter snare removal of acute middle cerebral artery thromboembolism: technical case report. Neurosurgery 2000;46:1529-31.

6 Kerber CW, Barr JD, Berger RM, et al. Snare retrieval of intracranial thrombus in patients with acute stroke. J Vasc Interv Radiol 2002;13:1268-74.

7 Wikholm G. Transarterial embolectomy in acute stroke. Am J Neuroradiol 2003;24:892-4.

8 Mayer TE, Hamann GF, Brueckmann H. Treatment of basilar artery embolism with mechanical extraction device: necessity of flow reversal. Stroke 2002;33:2232-5.

9 Yu W, Binder D, Foster-Barber A, et al. Endovascular embolectomy of acute basilar artery occlusion. Neurology 2003;61:1421-3.

10 Starkman S. Results of the Combined MERCI ${ }^{\circledR}$ I-II (mechanical embolus removal in cerebral ischemia) trials [abstract]. Stroke 2004;35:240.

11 Nedeltchev K, Remonda L, Do DD, et al. Acute stenting and thromboaspiration in basilar artery occlusions due to embolism from the dominating vertebral artery. Neuroradiology 2004;46:686-91.

12 Arnold M, Nedeltchev K, Schroth G, et al. Clinical and radiological predictors of recanalisation and outcome of 40 patients with acute basilar artery occlusion treated with intra-arterial thrombolysis. J Neurol Neurosurg Psychiatry 2004;75:857-62.

13 Eckert B, Kucinski T, Pfeiffer G, et al. Endovascular therapy of acute vertebrobasilar occlusion: early treatment onset as the most important factor. Cerebrovasc Dis 2002; 14:42-50.

14 Becker KJ, Monsien LH, Ulatowski J. Intraarterial thrombolysis in vertebrobasilar occlusion. Am J Neuroradiol 1996;17:255-62.

15 Brandt T, von Kummer R, Mueller-Kueppers M, et al. Thrombolytic therapy of acute basilar artery occlusion: variables affecting recanalization and outcome. Stroke 1996;27:875-81.

16 Cross DT, Moran CJ, Akins PT, et al. Collateral circulation and outcome after basilar artery thrombolysis. Am J Neuroradiol 1998;19:1557-63.

17 Cross DT, Moran JM, Akins PT, et al. Relationship between clot location and outcome after basilar artery thrombolysis. Am J Neuroradiol 1997; 18:1221-8.

18 Kucinski T, Koch C, Grzyska U. The predictive value of early CT and angiography for fatal hemispheric swelling in acute stroke. Am J Neuroradiol 1998; 19:839-46

19 Arnold M, Nedeltchev K, Mattle HP, et al. Intra-arterial thrombolysis in 24 consecutive patients with internal carotid artery T occlusions. J Neurol Neurosurg Psychiatry 2003;74:739-42.

20 Furlan A, Higashida R, Wechsler L, et al. Intra-arterial prourokinase for acute ischemic stroke. The PROACT II study. JAMA 1999;282:2003-11.

21 Gobin PY, Starkman S, Duckwiler GR, et al. MERCI 1: a phase 1 study of mechanical embolus removal in cerebral ischemia. Stroke 2004;35:2848-54.

22 Lindsberg PJ, Soinne L, Tatlisumak T, et al. Long-term outcome after intravenous thrombolysis of basilar artery occlusion. JAMA 2004;292: 1862-6. 